\title{
Radio Wave Propagation Scene Partitioning for High-Speed Rails
}

\author{
Bo Ai, Ruisi He, Zhangdui Zhong, Ke Guan, Binghao Chen, Pengyu Liu, and Yuanxuan Li
}

State Key Laboratory of Rail Traffic Control and Safety, Beijing Jiaotong University, Beijing 100044, China

Correspondence should be addressed to Bo Ai, aibo@ieee.org

Received 11 July 2012; Revised 20 August 2012; Accepted 5 September 2012

Academic Editor: César Briso Rodríguez

Copyright () 2012 Bo Ai et al. This is an open access article distributed under the Creative Commons Attribution License, which permits unrestricted use, distribution, and reproduction in any medium, provided the original work is properly cited.

Radio wave propagation scene partitioning is necessary for wireless channel modeling. As far as we know, there are no standards of scene partitioning for high-speed rail (HSR) scenarios, and therefore we propose the radio wave propagation scene partitioning scheme for HSR scenarios in this paper. Based on our measurements along the Wuhan-Guangzhou HSR, Zhengzhou-Xian passenger-dedicated line, Shijiazhuang-Taiyuan passenger-dedicated line, and Beijing-Tianjin intercity line in China, whose operation speeds are above $300 \mathrm{~km} / \mathrm{h}$, and based on the investigations on Beijing South Railway Station, Zhengzhou Railway Station, Wuhan Railway Station, Changsha Railway Station, Xian North Railway Station, Shijiazhuang North Railway Station, Taiyuan Railway Station, and Tianjin Railway Station, we obtain an overview of HSR propagation channels and record many valuable measurement data for HSR scenarios. On the basis of these measurements and investigations, we partitioned the HSR scene into twelve scenarios. Further work on theoretical analysis based on radio wave propagation mechanisms, such as reflection and diffraction, may lead us to develop the standard of radio wave propagation scene partitioning for HSR. Our work can also be used as a basis for the wireless channel modeling and the selection of some key techniques for HSR systems.

\section{Introduction}

Radio propagation environments may introduce multipath effects causing fading and channel time dispersion. Various propagation environments have different path loss and multipath effects, leading to the impossibility of radio wave propagation prediction in different propagation environment with the utilization of the same propagation channel model. Therefore, we should develop different wireless channel models according to radio propagation environments. That is to say, radio wave propagation scene portioning plays a very important role in wireless channel modeling. Scene partitioning is also the basis for the upper layer communication network design. Optimization with respect to radio wave propagation will greatly improve the planning of wireless networks for rails. Special railway structures such as cuttings, viaducts, and tunnels have a significant impact on propagation characteristics. However, these scenarios for high-speed rails (HSRs) have rarely been investigated, and few channel measurements have actually been carried out. Consequently, detailed and reasonable definitions for various scenarios in HSR are still missing. Therefore, a set of reasonable propagation scenarios for HSR environments needs to be defined so that statistical wireless channel models for HSR can be developed.

The main drawback of the current channel modeling approaches to railway communication is that the standard channel models used in the engineering implementation of HSR do not cover the special railway scenarios of cutting, viaducts, tunnels, and so on. For example, based on measurements obtained from the Zhengzhou-Xian passengerdedicated line, operating at speeds of around $350 \mathrm{~km} / \mathrm{h}$, we have found that the Hata model (which is used for path loss prediction) might result in about $17 \mathrm{~dB}$ errors for wireless network coverage prediction, as it does not include the diffraction loss caused by the cuttings along the rails [1]. The recently proposed WINNER model [2, 3] treats rail structures as one species with no distinguishing characteristics between them, which may be unreasonable. In addition, the working frequency of the WINNER model is from 2 to $6 \mathrm{GHz}$, which is not suitable for GSM for railway (GSM-R) wireless network operating at $930 \mathrm{MHz}$. 
This motivated us to carry out the research on radio wave propagation characteristics under the special scenarios for rails in order to obtain much more accurate path loss prediction results.

Several scene partitioning schemes for public wireless network communications are presented in Section 2. In Section 3, detailed descriptions about special scenarios in HSR are conducted. The scene partitioning scheme for HSR is proposed in Section 4, followed by the conclusions in Section 5.

\section{Overview of Scene Partitioning Schemes}

Several organizations and related standards should be mentioned when we refer to the scene partitioning. International Mobile Telecom System-2000 (IMT-2000) was proposed by International Telecommunication Union (ITU). It claims that [4] the purpose of defining distinct IMT-2000 radio operating environments is to identify scenarios that, from a radio perspective, may impose different requirements on the radio interface(s). It defines nine terrestrial scenarios and four satellite scenarios including business indoor, neighborhood indoor/outdoor, home, urban vehicular, urban pedestrian outdoor, rural outdoor, terrestrial aeronautical, fixed outdoor, local high bit rate environments, urban satellite, rural satellite, satellite fixed-mounted, and indoor satellite environments.

Universal Mobile Telecommunications System (UMTS) is developed by 3GPP. It claims that [5] a smaller set of radio propagation environments is defined which adequately span the overall range of possible environments. For practical reasons, these operating environments are an appropriate subset of the UMTS-operating environments described in Recommendation ITU-R M. 1034 [4].

WINNER project group in Europe was established in 2004. Based on UMTS and IMT-2000 scenario definitions, it defines four typical scenarios including in and around building, hot spot area, metropolitan, and rural scenarios. Eighteen detailed scenarios are defined on the basis of these four typical scenarios. The propagation scenarios listed above have been specified according to the requirements agreed commonly in the WINNER project [6]. The only one scenario appropriate for HSR defined in WINNER project is WINNER D2 model (rural moving network) [3]. However, the measurement environment for WINNER D2a is in European countries. These environments include no variable complicated HSR such as cuttings and viaducts. Moreover, as is mentioned in Section 1, the working frequency of WINNER D2a is at 2-6 GHz, which is not suitable for wireless network operating at $930 \mathrm{MHz}$.

Nowadays, more and more statistical wireless channel modeling approaches depend on Geographic Information System (GIS). Some GIS technology companieo define scenarios for wireless communications as well. These defined scenarios include inland water area, open wet area, open suburban, green land, forest, road, village, and tower.

Above all, the entire above-mentioned scene partitioning schemes include no special scenarios in HSR such as cuttings, viaducts, tunnels, and marshaling stations, which is not beneficial to wireless channel modeling for HSR. Therefore, it is necessary to establish the detailed scene partitioning scheme for HSR in order to improve the quality of dedicated wireless network planning and optimization.

\section{Special Scenarios for High-Speed Rails}

Based on our practical investigations on the ZhengzhouXian passenger-dedicated line, Wuhan-Guangzhou HSR, and some railway stations such as Beijing South Railway Station and Zhengzhou Railway Station, we obtained the valuable testing data for the HSR channels.

The actual measurements conditions are as follows $[7,8]$ : $930 \mathrm{MHz}$ narrowband measurements along the ZhengzhouXian HSR of China, using GSM-R base stations (BSs). The cross-polarization directional antennas of BSs positioned $10-20 \mathrm{~m}$ away from the track are utilized, with $17 \mathrm{dBi}$ gain, $43 \mathrm{dBm}$ TX power. The height of BS antenna varies in different scenarios, ranging from 20 to $60 \mathrm{~m}$. The omnidirectional receiver antennas are placed in the middle of the train, mounted on the top with the height of $30 \mathrm{~cm}$ above the train roof and $4 \mathrm{dBi}$ gain. The train moves at the speed up to $350 \mathrm{~km} / \mathrm{h}$. The samples are collected at $53 \mathrm{~cm}$ interval for large-scale analysis (the small scale effect is removed by averaging samples at the interval of $13 \mathrm{~m}$ ) and $10 \mathrm{~cm}$ interval for small scale analysis $[9,10]$. Note that our work uses the practical GSM-R network of HSR for measurement, at $930 \mathrm{MHz}$. Therefore, some of the channel parameters we present in the following may not be valid for other frequencies. However, our scene partitioning can be used in other communication systems for railways, such as GSM-R and long-term evolution for railway (LTE-R). In the following, we will describe the special propagation scenarios of HSR.

3.1. Viaducts. Viaduct is one of the most common scenarios in HSR (viaduct makes up $86.5 \%$ of the newly-opened Beijing Shanghai HSR of China).

Viaduct is a long bridge-like structure, typically a series of arches, carrying a railway across a valley or other uneven ground. In HSR constructions, it is difficult to lay the tracks on the uneven ground when the smoothness of rails is strictly required to ensure the high speed $(350 \mathrm{~km} / \mathrm{h})$ of the train. To overcome this problem, viaducts with a height of $10 \mathrm{~m}$ to $30 \mathrm{~m}$ are quite necessary, as is shown in Figure 1. Generally, the transmitter antennas are usually 20-30 m higher than the surface of the track, and the receiver antennas are mounted on top of the high-speed trains. Under this condition, few scatterers are higher than the viaduct, and the direct ray dominates with regard to the radio wave propagation, which makes viaduct a typical LOS propagation scenario [8]. We defined two categories of viaducts according to line-of-sight (LOS) and none-line-of-sight (NLOS) conditions.

3.1.1. Viaduct-1a. Viaduct-1a corresponds to the scenario that has some scatterers (such as trees and buildings) higher than the surface of the viaduct, most of which are located 


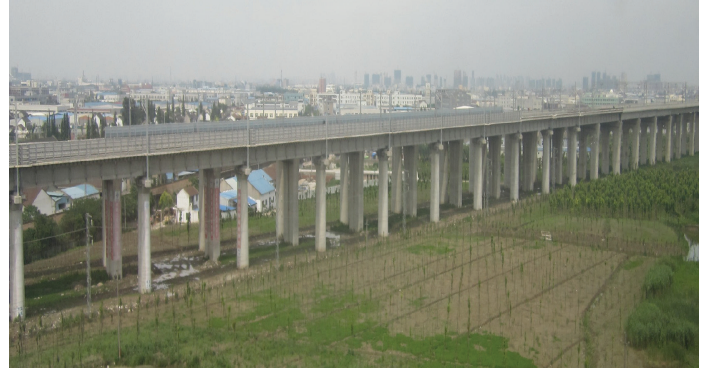

FIgURE 1: Viaduct scenario.

within a range of $50 \mathrm{~m}$ from the viaduct. These scatterers introduce rich reflection and scattering components, resulting in great severity of shadow fading. The stochastic changes of these scatters (such as the swing of the trees caused by wind) may also lead to the changes of the fading distribution.

3.1.2. Viaduct-1b. Viaduct- $1 \mathrm{~b}$ corresponds to the scenario that most scatterers, located within a range of $50 \mathrm{~m}$ from the viaduct, are lower than the surface of the viaduct. Under this condition, LOS is rarely blocked, and the direct path makes the greatest contribution to the propagation compared with other reflected and scattered paths. The effects of these scatterers (lower than the viaduct, or $50 \mathrm{~m}$ far from the viaduct) on propagation characteristics are negligible.

3.2. Cuttings. Cutting is a common scenario in HSR environments, which helps to ensure the smoothness of rails and high speed of the train operation [1]. It is used in HSR construction on uneven ground and to pass or "cut" through large obstacles such as hills. The cutting sides are usually covered with vegetation and reinforced concrete in case of subsidence. The forms of cutting can be either regular, where the steep walls on both sides of the rails have almost the same depths and slopes, or irregular owing to the locations of irregular hills or mountains along the line. This special structure of cutting creates a big "container," with rich reflection and scattering.

Cutting usually can be described with three parameters: crown width, bottom width, and depth of cutting. In Chinese HSR constructions, the crown width of cutting mostly ranges from 48 to $63 \mathrm{~m}$, while the bottom width ranges from 14 to $19 \mathrm{~m} \mathrm{[7].} \mathrm{The} \mathrm{depth} \mathrm{of} \mathrm{cutting} \mathrm{is} \mathrm{usually} 3-10 \mathrm{~m}$. These parameters can greatly measure the goodness of a cutting to be a container, for example, whether the cutting is too "wide" or "open" to hold enough multipath components.

The most common cutting is the regular deep cutting, where the steep walls on both sides of the rails have almost the same depths and slopes, as is shown in Figure 2. Under this condition, the receiver antenna is mostly lower than the roof of the cutting, leading to much more multipath components at the receiver. Moreover, the cross-bridges built over the cuttings lead to NLOS propagation in a short distance and may cause extra large-scale loss due to diffraction or other radio wave propagation mechanisms. Consequently, cutting has a significant impact on radio

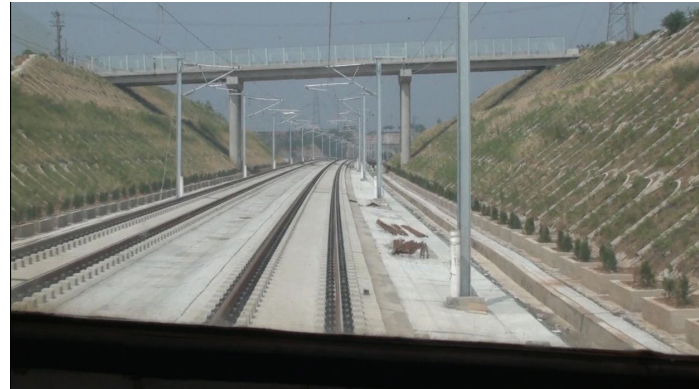

FIgURE 2: Cutting scenario.

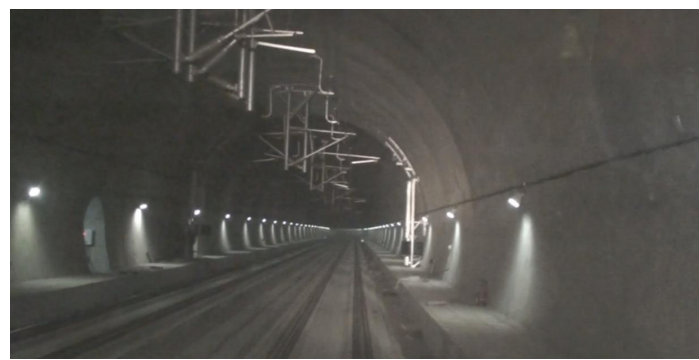

FIgURe 3: Tunnel scenario.

wave propagation characteristics and so can be disruptive to wireless communication.

3.3. Tunnels. Tunnel is an artificial underground passage, especially one built through a mountain in HSR environment, as is shown in Figure 3. The presence of tunnel ensures the high speed of train operation in rolling terrain. The sectional view of tunnel in HSR is usually vaulted or semicircle, with a height of 5-10 $\mathrm{m}$ and a width of $10-20 \mathrm{~m}$. The length of the tunnel in HSR mostly ranges from several to dozens of kilometers.

Generally, two main BSs are placed at the beginning and the end of the tunnel in HSR. Dependent on the length of the tunnel, several sub-BSs are placed inside the tunnel, installed in the wall. These sub-BSs help to provide great wireless coverage inside the tunnel. Due to the smooth walls and the close structure of the tunnel, there are rich reflections and scattering components inside the tunnel, which introduce the wave guide effect dominating the radio wave propagation inside the tunnel. This phenomenon makes the prediction of wireless signal in tunnel totally different from other propagation scenarios.

3.4. Railway Stations. Railway station is a railway facility where trains regularly stop to load or unload passengers. It generally consists of a platform next to the tracks and a depot providing related services such as ticket sales and waiting rooms. In the station scenario, the speed of the train is usually less than $80 \mathrm{~km} / \mathrm{h}$, while the speed of the crowd is $3-5 \mathrm{~km} / \mathrm{h}$. Due to the large number of users, high traffic requirements are expected in this environment. Moreover, the big awnings are usually utilized in stations to stop the rain from reaching the passengers and the trains, which may block 


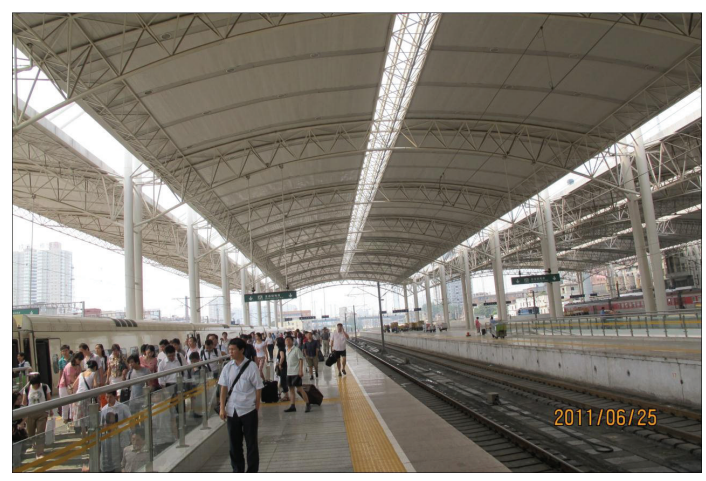

Figure 4: Medium- or small-sized station scenario.

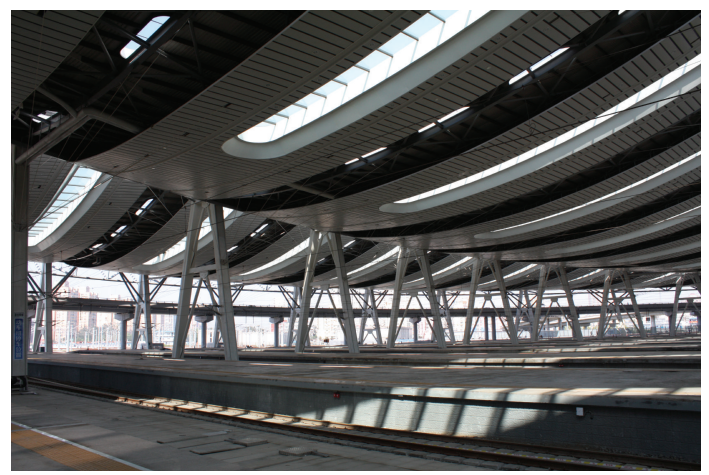

FIGURE 5: Large station scenario.

the LOS. Based on the capacity of the transportation, stations in HSR can be divided into three categories: medium- or small-sized station (4a), large station (4b), and marshaling station and container depot (4c).

3.4.1. Station-4a. Station-4a scenario indicates the medium or small-sized stations, as is shown in Figure 4. Mostly, there is not any awning on top of the trails, and the propagation could be both LOS and NLOS conditions. This scenario is similar to suburban environment. However, the passengers and platforms are usually close to the track so that the medium traffic requirements are expected in this environment.

3.4.2. Station- $4 b$. Station- $4 \mathrm{~b}$ scenario indicates the quite large and busy stations in terms of daily passenger throughput. These stations are used by an average of more than 60 thousand people, or 6500 trains per day, such as Beijing South Railway station, Guangzhou South Railway Station, and Xian North Railway Station. In Station-4b, there are usually big awnings on top of the rails, as is shown in Figure 5, making Station-4b similar to some indoor propagation scenarios $[6,7]$. The BSs are mostly located outside the awnings, sometimes inside the awnings. This special structure has a significant impact on radio wave propagation characteristics, especially when the train moves into or out of the railway station.

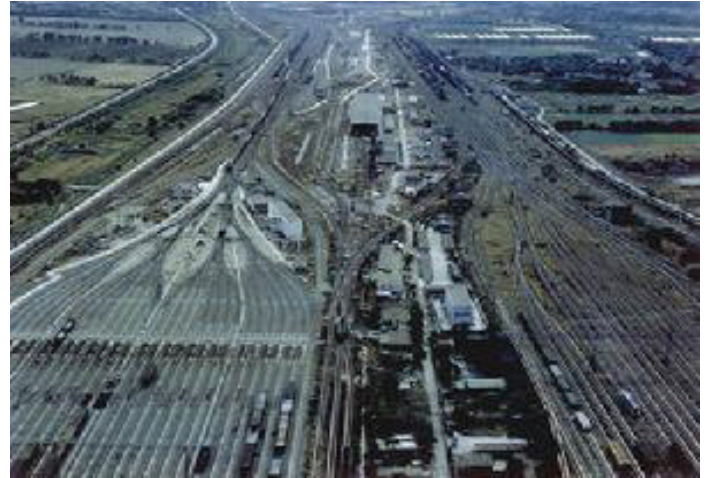

Figure 6: Marshaling station and container depot scenarios.

3.4.3. Station-4c. Station- $4 \mathrm{c}$ scenario indicates the marshaling stations and container depots, where the carriages are marshaled before traveling, or the train stops to load or unload freight, as is shown in Figure 6. In this scenario, the great traffic requirements of train controlling signal are highly expected. In addition, a number of metallic carriages result in complex multipath structure and the rich reflection and scattering components.

3.5. Combination Scenarios. Considering the complex environments along the HSR, several propagation scenarios may exist in one communication cell. This combination of the propagation scenarios is a challenging task for prediction of wireless signal. There are usually two categories of combination scenarios in HSR: tunnel group (11a), and cutting group (11b).

3.5.1. Combination Scenario-11a. Tunnel groups are widely present when the train passes through multimountain environment. In this terrain, the train will not stay in tunnel all the time, but frequently moves in and moves out of the tunnel. Under this condition, the transition areas are usually viaduct scenario. The frequent changes of the propagation scenario from tunnel to viaduct will greatly increase the severity of fading at the beginning or the end of the tunnel, resulting in poor communication quality.

3.5.2. Combination Scenario-11b. In cutting scenario, the depth of the cutting changes frequently. Sometimes, the steep walls on both sides may transitorily disappear, where the transition areas can be considered as the rural scenario. The frequent changes of scenario among deep cutting, low cutting, and rural can be quite disruptive to wireless communication, making the wireless signal prediction a great challenge.

3.6. In-Carriage. In-carriage scenario corresponds to the radio wave propagation used to provide personal communications for passengers with high quality of service. We define two categories of in-carriage scenarios in HSR: relay transmission (12a), and direct transmission (12b). 
TABLE 1: Radio wave propagation scene partitioning for HSR.

\begin{tabular}{|c|c|c|c|c|c|c|}
\hline Scenarios & Definitions & Sub scenarios & LOS/NLOS & $\begin{array}{l}\text { Speed } \\
(\mathrm{km} / \mathrm{h})\end{array}$ & $\begin{array}{l}\text { Special propagation } \\
\text { mechanisms }\end{array}$ & Notes \\
\hline \multirow{2}{*}{ S1 } & \multirow{2}{*}{ Viaduct } & Viaduct-1a & LOS & $0-350$ & & \\
\hline & & Viaduct-1b & LOS & $0-350$ & & \\
\hline S2 & Cutting & & LOS & $0-350$ & & \\
\hline S3 & Tunnel & & LOS & $0-250$ & Guide effect & \\
\hline \multirow{3}{*}{ S4 } & \multirow{3}{*}{ Station } & Station-4a: medium- or small-sized station & LOS/NLOS & $0-80$ & & \\
\hline & & Station- $4 \mathrm{~b}$ : large station & LOS/NLOS & $0-80$ & & \\
\hline & & $\begin{array}{l}\text { Station-4c: marshaling station and container } \\
\text { depot }\end{array}$ & LOS & $0-80$ & & \\
\hline \multirow{2}{*}{ S5 } & \multirow{2}{*}{ Water } & Water-5a: river and lake areas & LOS & $0-350$ & & \\
\hline & & Water-5b: sea area & LOS & $0-350$ & & \\
\hline S6 & Urban & & LOS & $0-350$ & & \\
\hline S7 & Suburban & & LOS & $0-350$ & & \\
\hline S8 & Rural & & LOS & $0-350$ & & \\
\hline \multirow[b]{2}{*}{ S9 } & \multirow[b]{2}{*}{ Mountain } & Mountain-9a: normal mountain & NLOS & $0-150$ & & \\
\hline & & Mountain-9b: far mountain & LOS & $0-350$ & & $\begin{array}{l}\text { Long delay } \\
\text { clutter }\end{array}$ \\
\hline S10 & Desert & & LOS & $0-350$ & Diffuse reflection & \\
\hline \multirow[b]{2}{*}{ S11 } & \multirow{2}{*}{$\begin{array}{l}\text { Combination } \\
\text { scenarios }\end{array}$} & Combination scenario-11a: tunnel group & LOS & $0-250$ & & \\
\hline & & Combination scenario-11b: cutting group & LOS & $0-350$ & & \\
\hline \multirow{2}{*}{ S12 } & \multirow{2}{*}{ In-carriage } & In-carriage-12a: relay transmission & LOS/NLOS & $0-5$ & & \\
\hline & & In-carriage-12b: direct transmission & NLOS & $0-350$ & Penetration loss & \\
\hline
\end{tabular}

TABLe 2: Predicted values of modeling parameters for HSR scenarios at $930 \mathrm{MHz}$.

\begin{tabular}{|c|c|c|c|c|c|}
\hline Scenarios & Definitions & Sub scenarios & $\begin{array}{l}\text { Path loss } \\
\text { exponent }\end{array}$ & $\begin{array}{l}\text { Standard deviation } \\
\text { of shadowing }(\mathrm{dB})\end{array}$ & $\begin{array}{l}\text { Fast fading } \\
\text { distribution }\end{array}$ \\
\hline \multirow{2}{*}{ S1 } & \multirow{2}{*}{ Viaduct } & Viaduct-1a & \multirow{2}{*}{$2-4$} & $3-4$ & \multirow{2}{*}{ Rice } \\
\hline & & Viaduct-1b & & $2-3$ & \\
\hline S2 & Cutting & & $2.5-4$ & $3-5$ & Rice \\
\hline S3 & Tunnel & & $1.8-3$ & $5-8$ & Rice \\
\hline \multirow[t]{2}{*}{ S4 } & \multirow[t]{2}{*}{ Station } & $\begin{array}{l}\text { Station-4a: medium- or small-sized station } \\
\text { Station-4b: large station }\end{array}$ & $3-5$ & $3-5$ & Rice/Rayleigh \\
\hline & & Station-4c: marshaling station and container depot & $2-4$ & $2-3$ & Rice \\
\hline \multirow{2}{*}{ S5 } & \multirow{2}{*}{ Water } & Water-5a: river and lake areas & \multirow{2}{*}{$2-4$} & \multirow{2}{*}{$2-3$} & \multirow{2}{*}{ Rice } \\
\hline & & Water-5b: sea area & & & \\
\hline S6 & Urban & & $4-7$ & $3-5$ & Rice \\
\hline S7 & Suburban & & $3-5$ & $2-3$ & Rice \\
\hline S8 & Rural & & $2-5$ & $2-3$ & Rice \\
\hline \multirow{2}{*}{ S9 } & \multirow{2}{*}{ Mountain } & Mountain-9a: normal mountain & $5-7$ & $3-5$ & Rayleigh \\
\hline & & Mountain-9b: far mountain & $3-5$ & $2-6$ & Rice \\
\hline S10 & Desert & & $2-4$ & $2-3$ & Rice \\
\hline \multirow{2}{*}{ S11 } & \multirow{2}{*}{$\begin{array}{l}\text { Combination } \\
\text { scenarios }\end{array}$} & Combination scenario-11a: tunnel group & \multirow[t]{2}{*}{$3-7$} & \multirow[t]{2}{*}{$5-8$} & \multirow[t]{2}{*}{ Rice } \\
\hline & & Combination scenario-11b: cutting group & & & \\
\hline \multirow[b]{2}{*}{ S12 } & \multirow[b]{2}{*}{ In-carriage } & In-carriage-12a: relay transmission & $1.5-5$ & $3-5$ & Rice/Rayleigh \\
\hline & & In-carriage-12b: direct transmission & $5-8$ & $4-7$ & Rayleigh \\
\hline
\end{tabular}




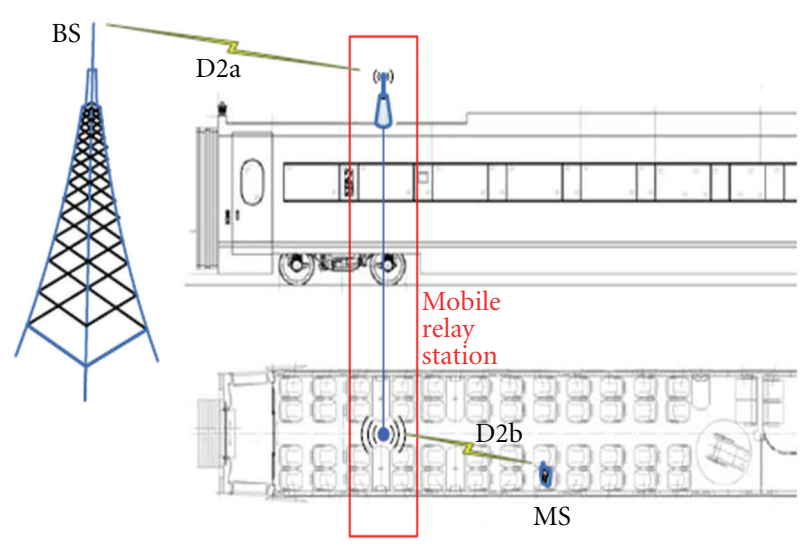

FIgURE 7: Relay transmission scenario in [3].

3.6.1. In-Carriage-12a. Relay transmission occurs in carriages of high-speed trains where wireless coverage is provided by the so-called moving relay stations which can be mounted to the ceiling [3], as is shown in Figure 7. Note that the link between the BS and the moving train is usually a LOS wireless link whose propagation characteristics are represented by other mentioned HSR propagation scenarios. Moreover, due to the great penetration loss of the carriage, the change of the environments outside the train has a negligible effect on radio wave propagation inside the train, and the propagation inside the carriage can be covered with the models for some typical indoor channels.

3.6.2. In-Carriage-12b. Direct transmission indicates the scenario that uses the wireless link between the BS and the user inside the carriage to provide the high-quality communications. Under this condition, the penetration loss of the carriage has a great effect on radio wave propagation. The wireless link between the BS and the moving train, together with the link inside the carriage and a reasonable value of the penetration loss, can be used to predict radio wave propagation in this scenario.

\section{Scene Partitioning Scheme for High-Speed Rails}

The proposed radio wave propagation scene partitioning scheme is presented in Table 1 . The corresponding modeling parameters for each scenario are shown in Table 2. Detailed description and theoretical analysis based on reflection, scattering, and diffraction propagation mechanism will be given in our future work.

For the proposed scene partitioning scheme, we take comprehensive consideration of three categories attributes. The first one is physical attribute, which means variation of radio wave propagation mechanism between $\mathrm{BS}$ and mobile users, for example, direct wave and reflection wave, lineof-sight (LOS)/non-line-of-sight (NLOS), and the variation of multipath structure. Such physical attributes may lead to such special scenarios in HSR such as viaducts, cuttings, and tunnels. This attribute is clearly unfolded in Table 1.
The second one is user attribute, which is related with the user requirements of the provided services. It mainly takes the factor of transmission rate and moving speed into consideration. This attribute is unfolded in the scene partitioning scheme as the moving speed of users.

The third one is related with coverage of wireless network. It considers various wireless network covering approaches. For example, ribbon covering approach is commonly adopted along the rails currently. This attribute is unfolded in the scene partitioning scheme as the scene of marshaling stations. The traffic volume in railway marshaling station is much higher than that of the ordinary railway stations.

In accordance with the testing data, the scenarios are appropriate for $930 \mathrm{MHz}$ working frequency. Note that the 10th scenario-desert-appears in Taiyuan-Yinchuan railway in China.

Note that the modeling parameters for scenarios S1 and S2 are based on our previous research results of $[7,8,11,12]$. The parameters for scenario S3 are based on the results of $[13,14]$. The parameters for scenarios S4, S6, S7, S8, and S9 are predicted based on our previous research results of [15]. The parameters for scenario S12 are based on the results of [6]. Moreover, the modeling parameters for each scenario are just the prediction values. Accurate parameters values could be obtained after accurate channel modeling.

\section{Conclusions}

Up till now, there is no any radio wave propagation scene partitioning scheme for HSR environments, which contains many special propagation scenarios, such as viaducts, cuttings, tunnels, and marshaling stations. Scene partitioning is very useful for wireless channel modeling, which is the basis for BS location, wireless network planning, and optimization. Only with the scene partitioning for HSR, the accurate path loss prediction models can be developed, which are the fundamental basis of wireless link budget and the basis of the position determination of the base stations for HSR network. In this paper, a series of propagation scenarios of HSR is reviewed based on the practical channel measurements in China, and the scene partitioning scheme is proposed. The results can be used for the propagation channel characterization in HSR environments. Our future work will focus on the theoretical analysis of these scenarios through such propagation mechanisms as reflection, diffraction and scattering. Corresponding wireless channel models for HSR on the basis of the scene partitioning will be studied as well. We will also pay attention to other working frequencies which could be used for railway communications in the future.

\section{Acknowledgment}

The authors are grateful to Hong Wei, Jing-hui Lu, Zimu Cheng, and other members of radio wave propagation and wireless channel modeling research group in Beijing Jiaotong University. They also express their many thanks 
for the supports from the National Science Foundation of China under Grant 61222105, the Beijing Municipal Natural Science Foundation under Grant 4112048, the Program for New Century Excellent Talents in University of China under Grant NCET-09-0206, the NSFC under Grant 60830001, the Fundamental Research Funds for the Central Universities under Grant 2010JBZ008, and the Key Project of State Key Lab under Grant no. RCS2011ZZ008.

\section{References}

[1] B. Ai, "Interview," Electronics Letters, vol. 47, no. 21, article $1158,2011$.

[2] WINNER Group, "IST-4-027756 WINNER II D1. 1. 1 V1. 1, WINNER II Interim channel models,” February 2007.

[3] WINNER Group and IST-WINNER II Deliverable 1. 1. 2 v. 1. 2, "WINNER II channel models," Tech. Rep. IST-WINNER2, 2008.

[4] I. T. U. . Rec, ITU-R M: 1034-1, Requirements for the radio interface(s) for international mobile telecommunications2000, (IMT-2000).

[5] ETSI, TR 101112 v3. 2. 0, Universal Mobile Telecommunications Systems (UMTS): selection procedures for the choice of radio transmission technologies of the UMTS, 1998.

[6] WINNER Group, "System assessment criteria specification," IST-2003-507581. WINNER, July 2004.

[7] R. He, Z. Zhong, B. Ai, and J. Ding, "Propagation measurements and analysis for high-speed railway cutting scenario," Electronics Letters, vol. 47, no. 21, pp. 1167-1168, 2011.

[8] R. He, Z. Zhong, B. Ai, and J. Ding, "An empirical path loss model and fading analysis for high-speed railway viaduct scenarios," IEEE Antennas and Wireless Propagation Letters, vol. 10, pp. 808-812, 2011.

[9] W. C. Y. Lee, "Estimate of local average power of a mobile radio signal," IEEE Transactions on Vehicular Technology, vol. 34, no. 1, pp. 22-27, 1985.

[10] B. Ai, Z. D. Zhong, G. Zhu, and M. Zhao, "Novel statistical criteria for local mean power estimation in wireless coverage perdiction," IET Microwaves, Antennas and Propagation, vol. 5, no. 5, pp. 596-604, 2011.

[11] H. Wei, Z. Zhong, L. Xiong, B. Ai, and R. He, "Study on the shadow fading characteristic in viaduct scenario of the highspeed railway," in Proceedings of the IEEE 6th International ICST Conference on Communications and Networking in China (CHINACOM), pp. 1216-1220, Haerbin, China, August 2011.

[12] R. He, Z. Zhong, B. Ai, and J. Ding, "Measurements and analysis of short-term fading behavior for high-speed rail viaduct scenario," in Proceedings of the IEEE ICC, pp. 1-5, Ottawa, Canada, 2012.

[13] C. Nerguizian, C. L. Despins, S. Affès, and M. Djadel, "Radiochannel characterization of an underground mine at $2.4 \mathrm{GHz}$," IEEE Transactions on Wireless Communications, vol. 4, no. 5, pp. 2441-2453, 2005.

[14] Y. P. Zhang, "A hybrid model for propagation loss prediction in tunnels," in Proceedings of the Millennium Conference on Antennas and Propagation, Davos, Switzerland, 2000.

[15] R. He, Z. Zhong, and B. Ai, "Path loss measurements and analysis for high-speed railway viaduct scene," in Proceedings of the 6th International Wireless Communications and Mobile Computing Conference (IWCMC'10), pp. 266-270, Caen, France, July 2010. 

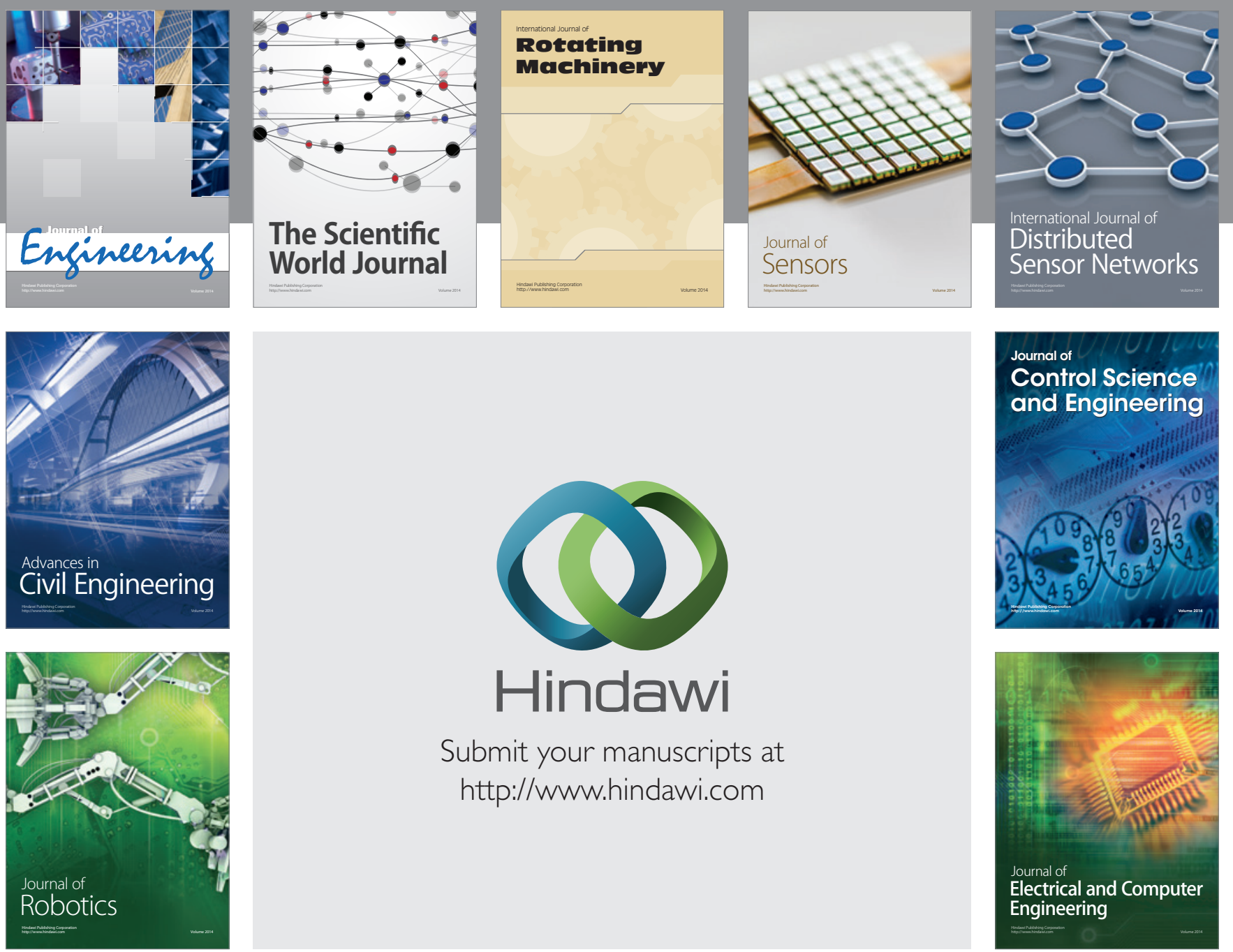

Submit your manuscripts at

http://www.hindawi.com
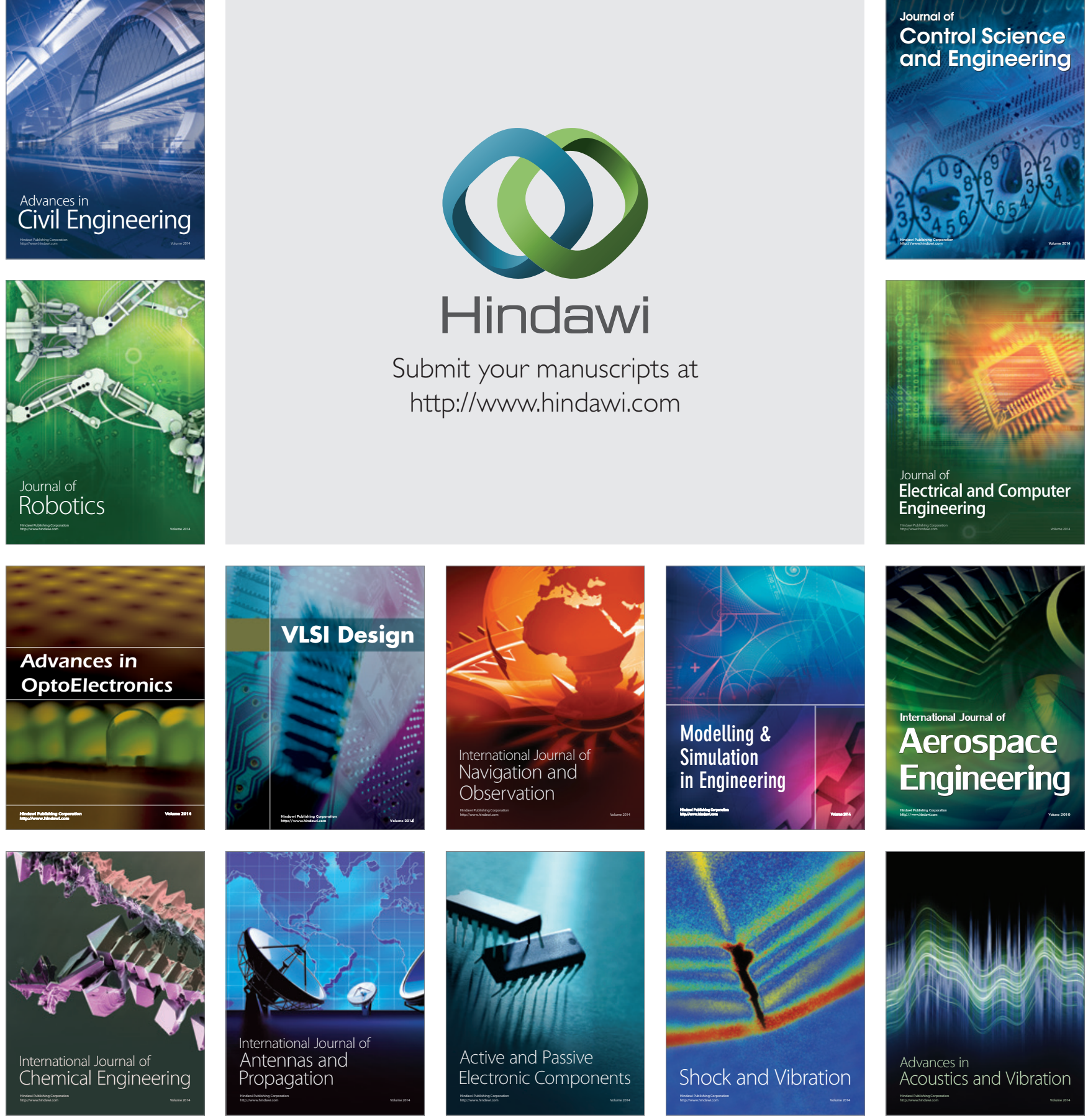